\title{
Use of array-based technology in the practice of medical genetics
}

\author{
Melanie Manning, $M D^{1,2}$, and Louanne Hudgins, $M D^{2}$
}

Key Words: mental retardation, malformations, array comparative genomic hybridization, cytogenetics

\begin{abstract}
Disclaimer: This guideline is designed primarily as an educational resource for health care providers to help them provide quality medical genetic services. Adherence to this guideline does not necessarily ensure a successful medical outcome. This guideline should not be considered inclusive of all proper procedures and tests or exclusive of other procedures and tests that are reasonably directed to obtaining the same results. In determining the propriety of any specific procedure or test, the geneticist should apply his or her own professional judgment to the specific clinical circumstances presented by the individual patient or specimen. It may be prudent, however, to document in the patient's record the rationale for any significant deviation from this guideline.
\end{abstract}

\section{ABSTRACT}

Mental retardation affects approximately $3 \%$ of the population, and the background birth defect rate is $3 \%$ to $4 \%$. An underlying cause is identified less than $50 \%$ of the time. In the cases in which a cause is determined, a chromosomal anomaly is the cause in up to $40 \%$. Laboratory evaluation routinely includes high-resolution karyotyping, subtelomeric fluorescence in situ hybridization analysis, and targeted fluorescence in situ hybridization analysis depending on the clinical features. There are technical limitations to these techniques, however. For example, anomalies less than 2 to $3 \mathrm{Mb}$ in size are undetectable by karyotype, and subtelomeric fluorescence in situ hybridization analysis is a labor-intensive analysis with a relatively low yield. With completion of the Human Genome Project, diagnostic testing is moving toward the use of DNAbased techniques such as comparative genomic hybridization microarray analysis or array comparative genomic hybridization. Although this technology has been used in the evaluation of tumors and cancer patients in the past, it is now being applied in the assessment of patients demonstrating idiopathic mental retardation or developmental delay, dysmorphic features, congenital anomalies, and spontaneous abortions. As with other well-developed cytogenetic studies, there are technical limitations to array comparative genomic hybridization that must be acknowledged and addressed before its widespread use. A variety of array-based technologies are now available on a clinical basis. We discuss the utility and limitations of using this technology in the evaluation of individuals with

From the Departments of ${ }^{1}$ Pathology and ${ }^{2}$ Pediatrics, Stanford University School of Medicine, Stanford, California.

Approved by the Board of Directors, March 21, 2007. Go to www.geneticsinmedicine.org for a printable copy of this document

American College of Medical Genetics, 9650 Rockville Pike, Bethesda, MD 20814-3998.

Disclosure: The authors declare no conflict of interest.

DOI: 10.1097/GIM.0b013e31814cec3a mental retardation and malformations, citing the existing literature.

\section{INTRODUCTION}

Conventional cytogenetic techniques [high-resolution GTG banding, subtelomeric fluorescence in situ hybridization (FISH), targeted FISH] play an important role in the evaluation of an individual with mental retardation or developmental delay, congenital anomalies, and dysmorphic features. However, there are technical limitations to these tests. For example, chromosome analysis cannot detect anomalies that are smaller than 2 to $3 \mathrm{Mb}$, targeted FISH is performed on a limited basis based on phenotype, and subtelomeric FISH is labor intensive for the relatively low yield of abnormalities detected. ${ }^{1}$

\section{DEFINITIONS}

Because gene dose variations are the underlying cause of many genetic conditions (e.g., Down syndrome, Prader-Willi syndrome), detection and mapping of gene copy number abnormalities have proven to be important in associating aberrations with disease phenotype and for localizing critical genes. ${ }^{2}$ Molecular cytogenetic techniques, such as comparative genomic hybridization (CGH), were developed for genomewide screening for chromosomal copy number variations in a single experiment. ${ }^{2,3}$ With this technique, now termed conventional CGH, patient and reference genomic DNA samples are differentially labeled with fluorescent dyes and hybridized to slides containing normal human metaphase chromosomes. DNA from both sources compete for their respective positions on the array. Computer analysis of the fluorescent signals determines overall gains and losses of DNA sequences across the genome. ${ }^{4}$ Because metaphase chromosomes were used for hybridization of sample and reference DNA, the resolution of conventional CGH was only 3 to $10 \mathrm{Mb}^{2}$, similar to that of high-resolution karyotyping. A substantial increase in resolu- 
tion came with development of CGH microarrays [array CGH $(\mathrm{aCGH})] \cdot{ }^{2,5}$ With aCGH, metaphase chromosomes are replaced by cloned [e.g., bacterial artificial chromosomes (BACs)] or synthesized [e.g., oligonucleotides (oligos)] DNA fragments across the genome that are immobilized on a glass surface, the exact chromosomal locus of which is known. ${ }^{4,6}$ Sample DNA and control DNA are hybridized to the DNA fragments spotted onto the array, and copy number variations are measured by computer analysis of the differences in hybridization pattern intensities. ${ }^{4}$ The resolution of aCGH is limited only by the size and distance between the BACs or oligos spotted onto the array. ${ }^{7}$

At this time, there are two types of array platforms being offered clinically: the constitutional or targeted array and the whole-genome array. The targeted array has a limited number of BAC clones corresponding to regions thought to be clinically significant (e.g., subtelomeric regions) or encompassing commonly described chromosomal alterations (e.g., microdeletion syndromes). ${ }^{3,8}$

Whole-genome arrays have now been developed that, as the name implies, have wider coverage over the human genome. One of the first whole-genome arrays was constructed by Snijders et al. ${ }^{6}$ and contained approximately 2400 BAC clones distributed over the genome. Newer arrays contain even more $\mathrm{BAC}$ clones, with lengths ranging from 60 to $200 \mathrm{~kb}$ that are spaced at intervals of approximately one clone per $1 \mathrm{Mb}$. Higher resolution is provided by these denser arrays. However, it is somewhat arbitrary coverage and clinically significant loci may not be included. Oligo arrays provide even greater resolution with thousands of oligomer probes placed at various spacings across the genome. Array design will dictate the ability to accurately delineate mutation intervals and the size of the DNA copy number changes that can be detected.

\section{UTILITY}

Until recently, microarrays have been designed for and used in the research arena. Arrays for individual chromosomes, telomeric regions, specific chromosome regions, and the whole genome have provided information on DNA gains or losses with unprecedented resolution. ${ }^{3}$ As the technology moves toward the clinical setting, the most appropriate use is being debated.

Currently, clinical availability of aCGH testing is basically limited to BAC clone targeted arrays. Several laboratories around the country now offer testing that is listed in GeneTests. ${ }^{9}$ In the near future, higher definition whole genome BAC arrays will be more widely available and will likely augment or replace the targeted array. Also on the horizon and approaching more widespread clinical availability are the oligo arrays. Because these arrays are technically easier to use, are higher resolution $(35 \mathrm{~kb})$, and can delineate mutation intervals with greater accuracy, oligo array technology may be the preferred technology in the future.

The scope of use of aCGH has broadened from the cancer research and diagnostic process ${ }^{10}$ to include evaluation of pa- tients with developmental issues and/or malformations as well as products of conception samples. In addition to individual case reports, case series of aCGH use are now being reported in the literature. Reports of results from targeted and whole-genome $\mathrm{BAC}^{7,11-16}$ and oligo ${ }^{17,18}$ arrays utilized in the evaluation of patients with mental retardation/developmental delay and dysmorphic features/congenital malformations began appearing in the medical literature in 2003. Study sample sizes have ranged from 8 to 1500 patients. The largest case series thus far include two reports by Menten et al..$^{15}$ and Shaffer et al. ${ }^{16}$ in which the authors report results for 140 and 1500 cases, respectively. However, other studies had substantially fewer patients ranging from 8 to $50.7^{7,11-14,17,19}$ The reported de novo DNA copy number change rate ranged from $8 \%$ to $40 \%$. There are limitations to all the studies published thus far, including limited clinical information and the lack of control studies.

In addition to new and presumed significant chromosomal changes, several of the studies demonstrated that the arrays could reliably detect previously described chromosomal abnormalities identified by standard cytogenetic techniques. ${ }^{20}$ Studies have also reported the detection of chromosomal mosaicism in the range of $3 \%$ to $20 \%$ in patients. ${ }^{15,16,20}$ Reports of aCGH identifying cytogenetic abnormalities missed by routine cytogenetic analyses are also appearing in the literature and further support the sensitivity of aCGH. ${ }^{16,20}$

In addition to using aCGH in the evaluation of patients with phenotypes suggestive of a chromosome abnormality (mental retardation, dysmorphic features, congenital anomalies), arrays have also been applied in the assessment of spontaneous miscarriage or abortion specimens and fetuses with multiple anomalies. ${ }^{21-23}$ Schaeffer et al. ${ }^{21}$ used a targeted array in their retrospective study, and, in addition to the anomalies detected by routine cytogenetic testing, aCGH identified four new abnormalities. However, the significance of the abnormalities (e.g., interstitial deletion of 9p21 in a trisomy 13 sample) was not addressed, and parental comparisons were not made.

The efficacy of applying microarray CGH technology to prenatal amniotic fluid and chorionic villus samples was examined by Rickman et al. ${ }^{24}$ In this study, 30 cultured pre-/postnatal samples with microscopically confirmed unbalanced rearrangements were examined using a custom, 600 large insert clone (BAC, PAC) array and a $1-\mathrm{Mb}$ resolution array. The customized array was designed to concentrate clones in areas of clinical significance with dense representation across common microdeletion syndrome regions. A lower representation (approximately one clone per $10 \mathrm{Mb}$ ) was used over the remainder of the genome. The custom prenatal array detected 29 of 30 abnormalities, but the 1-Mb array only found 22 of 30 of the abnormalities. Both arrays failed to detect a triploid karyotype. Uncultured samples, from as little as $1 \mathrm{~mL}$ of uncultured amniotic fluid and a single small fragment of chorionic villus, also showed results on the arrays that were concordant with cultured samples. The authors stated that their study demonstrates the potential for aCGH to replace conventional cytogenetics in a majority of prenatal diagnosis cases. However, they did point out limitations of the technology such as the inability 
of an array to detect polyploidy and balanced chromosomal rearrangements. They also noted that higher density arrays are likely to generate abnormalities that will be difficult to interpret in the context of copy number variations identified with the array. Shimokawa et al.22 echoed this concern by questioning the ability at this point to be able to comment on the phenotypic effects of dose differences in a few clones. Would these changes be significant clinically? These are questions that cannot be answered at this stage of array use. If applied in a prenatal setting, the additional stress and anxiety generated with this type of uncertainty will be difficult for clinicians and genetic counselors to address. Rickman et al. ${ }^{24}$ suggested that a targeted (limited) prenatal array be designed that excludes known regions of copy number changes associated with mild phenotypic abnormalities to minimize difficulties with interpretation. However, adding clones to an array (i.e., to detect microdeletion syndromes) that may not show an increased incidence in the majority of women undergoing prenatal testing for maternal age raises the question of who should now be offered this type of testing.

\section{CONCERNS}

\section{Copy number variants}

As banded chromosome analysis became a standard part of the evaluation of an individual with mental retardation or congenital anomalies, it became apparent that certain regions of the karyotype showed normal variation within the human population (e.g., pericentric inversion of chromosome 9, qh regions of chromosomes 1, 9, 16, Y). Further definition of the human genome through the Human Genome Project and technology such as aCGH have identified another form of variation in the DNA: large-scale copy number variants (CNVs). ${ }^{25,26}$ These are currently being catalogued in databases such as the Database of Genomic Variants (public Web site: http://projects.tcag.ca/variation)and DECIPHER. All DNA copy number alterations are being compiled, both those thought to be clinically relevant as well as changes shown to be polymorphisms or variants. The extent to which these CNVs are present throughout the genome and what exact role they play in human genetic variation are yet to be determined and is an area of active research (reviewed in Feuk et al. ${ }^{27}$ ). At this time, if an abnormality is detected, the answer as to whether it is a pathologic change may be difficult to determine with questions about CNVs still unanswered. Although parental analyses in such cases may be helpful, additional information regarding CNVs and polymorphisms may be gained from general population screening with microarrays. To date, a project of this magnitude has not been undertaken.

Historically, to determine whether a chromosome abnormality was an inherited or de novo finding, parental samples were examined. This protocol will be followed with the application of aCGH. Specific BAC clones are used to verify array results on patients. Parental samples are then examined for any abnormality detected. In some instances, arrays may also be required on parents to clarify breakpoints or confirm CNVs.
Following these procedures will unquestionably add additional costs to an already expensive test. However, until such time as a comprehensive $\mathrm{CNV}$ and polymorphism database is compiled that can be used for result comparison, interpretation of aCGH results will still require adjunctive testing for clarification as is routinely done in cytogenetics laboratories at this time.

\section{Cost}

Not surprisingly, aCGH testing is currently an expensive test, with estimates ranging from $\$ 1500$ to $\$ 1800$. However, if one assumes the cost of standard karyotyping, with subtelomeric FISH and possibly locus-specific FISH, the total cost for this kind of workup exceeds that currently being charged for aCGH. As with other highly technical applications, prices tend to decrease as techniques are honed and production costs drop. For the foreseeable future, the cost of performing aCGH on a patient will be similar to that charged for subtelomeric FISH analysis.

\section{Standardization}

An issue that should be considered before the widespread availability of aCGH is the lack of standardization for array production, analysis, and reporting of results. Arrays in use at this time are mostly "home brews" designed by individual laboratories performing the test. Computer analysis programs can also be customized for particular arrays. The American College of Medical Genetics is actively addressing the issues regarding laboratory guidelines (see page 654 in this issue). In addition, the ISCN $2005{ }^{28}$ provides nomenclature for reporting microarray results to help standardize the reporting of chromosome abnormalities and normal results obtained from this new technology.

\section{SUMMARY}

aCGH analysis is proving to be a sensitive and reliable tool in detecting submicroscopic chromosomal abnormalities that are undetectable by current cytogenetic testing methodologies. Reports to date are consistently quoting an abnormality rate between $8 \%$ and $20 \%$, although larger numbers of patients need to be studied with these techniques. The discovery of novel chromosome abnormalities and newly identified syndromes ${ }^{29-32}$ will surely increase, and identification of important chromosomal loci will further direct research into genes crucial for normal growth and development. However, there are still some issues (e.g., CNVs, standardization of reporting) that need to be addressed as aCGH is offered clinically on a widespread basis. Because this technology may identify DNA copy number changes of uncertain clinical significance, its use in the prenatal setting should be approached with caution.

\section{Recommendations:}

1. At the present time, microarray CGH may be used as an adjunct to standard cytogenetic testing (including targeted FISH for specific 
microdeletion/duplication syndromes) in the evaluation of a patient with mental retardation and/or congenital anomalies. Financial limitations, availability of parents for testing, and the possible ambiguity of results should all be considered.

2. Microarray CGH should not be used as a first-tier test in prenatal diagnosis. Limited use of the targeted array may be helpful in the evaluation of fetuses with structural anomalies and normal chromosome analysis or of marker chromosomes. ${ }^{33}$

\section{References}

1. Saal HM, Bao L, Schorry EK, Hopkin RJ, et al. Paucity of primary identification of new cryptic subtelomeric rearrangements with subtelomere FISH: a three year retrospective analysis. Proc Greenwood Genet Cent 2006;25:99-100.

2. Pinkel D, Segraves R, Sudar D, Clark S, et al. High resolution analysis of DNA copy-number variation using comparative genomic hybridization to microarrays. Nat Genet 1998;20:207-2111.

3. Bejjani BA, Saleki R, Ballif BC, Rorem EA, et al. Use of targeted array-based CGH for the clinical diagnosis of chromosomal imbalance: is less more? Am J Med Genet 2005;134A:259-267.

4. Oostlander AE, Meijer GA, Ylstra B. Microarray-based comparative genomic hybridization and its applications in human genetics. Clin Genet 2004;66:488-495.

5. Solinas-Toldo S, Lampel S, Stilgenbauer S, Nickolenko J, et al. Matrix-based comparative genomic hybridization: biochips to screen for genomic imbalances. Genes Chromosomes Cancer 1997;20:399-407.

6. Snijders AM, Nowak N, Segraves R, Blackwood S, et al. Assembly of microarrays for genome-wide measurements of DNA copy number. Nat Genet 2001;29:263-264.

7. Tyson C, Harvard C, Locker R, Friedman JM, et al. Submicroscopic deletions and duplications in individuals with intellectual disability detected by array-CGH. Am J Med Genet 2005;139A:173-185.

8. Cheung SW, Shaw CA, Yu W, Li J, et al. Development and validation of a CGH microarray for clinical cytogenetic diagnosis. Genet Med 2005;7:422-432.

9. Gene Tests: Medical Genetics Information Resource [database online]. Seattle, WA: University of Washington;2007. Available at:http://www.genetests.org.

10. Albertson DG, Pinkel D. Genomic microarrays in human genetic disease and cancer. Hum Mol Genet 2003;12:R145-R152.

11. Vissers LELM, de Vries BBA, Osoegawa K, Janssen IM, et al. Array-based comparative genomic hybridization for the genomewide detection of submicroscopic chromosomal abnormalities. Am J Hum Genet 2003;73:1261-1270.

12. Shaw-Smith C, Redon R, Rickman L, Rio M, et al. Microarray based comparative genomic hybridization (array-CGH) detects submicroscopic chromosomal deletions and duplications in patients with learning disability/mental retardation and dysmorphic features. J Med Genet 2004;41:241-248.

13. Schoumans J, Ruivenkamp C, Holmberg E, Kyllerman M, et al. Detection of chromosomal imbalances in children with idiopathic mental retardation by array based comparative genomic hybridisation (array-CGH). J Med Genet 2005;42:699-705.

14. Bar-Shira A, Rosner G, Rosner S, Goldstein M, et al. Array-based comparative genome hybridization in clinical genetics. Pediatr Res 2006;60:353-358.

15. Menten B, Maas N, Thienpont B, Buysse K, et al. Emerging patterns of cryptic chromosomal imbalance in patients with idiopathic mental retardation and multi- ple congenital anomalies: a new series of 140 patients and review of published reports. J Med Genet 2006;43:625-633.

16. Shaffer LG, Kashork CD, Saleki R, Rorem E, et al. Targeted genomic microarray analysis for identification of chromosome abnormalities in 1500 consecutive clinical cases. J Pediatr 2006;149:98-102.

17. Ming JE, Geiger E, James AC, Ciprero KL, et al. Rapid detection of submicroscopic chromosomal rearrangements in children with multiple congenital anomalies using high density oligonucleotide arrays. Hum Mutat 2006;27:467-473.

18. Friedman JM, Baross A, Delaney AD, Ally A, et al. Oligonucleotide microarray analysis of genomic imbalance in children with mental retardation. Am J Hum Genet 2006;79:500-513.

19. Aradhya S, Manning MA, Splendore A, Cherry AM. Whole-genome array-CGH identifies novel contiguous gene deletions and duplications associated with developmental delay, mental retardation, and dysmorphic features. J Med Genet A 2007; 143:1431-1441.

20. Ballif BC, Rorem EA, Sundin K, Lincicum M, et al. Detection of low-level mosaicism by array CGH in routine diagnostic specimens. Am J Med Genet 2006;140A:27572767.

21. Schaeffer AJ, Chung J, Heretis K, Wong A, et al. Comparative genomic hybridization-array analysis enhances the detection of aneuploides and submicroscopic imbalances in spontaneous miscarriages. Am J Hum Genet 2004;74:1168-1174.

22. Shimokawa O, Harada N, Miyake N, Satoh K, et al. Array comparative genomic hybridization analysis in first-trimester spontaneous abortions with 'normal' karyotypes. Am J Med Genet Part A 2006;140A:1931-1935.

23. Le Caignec C, Boceno M, Saugier-Veber P, Jacquemont S, et al. Detection of genomic imbalances by array based comparative genomic hybridisation in fetuses with multiple malformations. J Med Genet 2005;42:121-128.

24. Rickman L, Fiegler H, Shaw-Smith C, Nash R, et al. Prenatal detection of unbalanced chromosomal rearrangements by array CGH. J Med Genet 2006;43:353-361.

25. Iafrate AJ, Feuk L, Rivera M, Listewnik ML, et al. Detection of large-scale variation in the human genome. Nat Genet 2004;36:949-951.

26. Sebat J, Lakshmi B, Troge J, Alexander J, et al. Large-scale copy number polymorphism in the human genome. Science 2004;305:525-528.

27. Feuk L, Marshall CR, Wintle RF, Scherer SW. Structural variants: changing the landscape of chromosomes and design of disease studies. Hum Mol Genet 2006;15: R57-R66.

28. Shaffer LG, Tommerup N, editors. ISCN 2005. Basel, Switzerland: Karger, 2005.

29. Koolen DA, Vissers LELM, Pfundt R, de Leeuw N, et al. A new chromosome $17 \mathrm{q} 21.31$ microdeletion syndrome associated with a common inversion polymorphism. Nat Genet 2006;38:999-1001.

30. Shaw-Smith C, Pittman AM, Willatt L, Martin H, et al. Microdeletion encompassing MAPT at chromosome $17 \mathrm{q} 21.3$ is associated with developmental delay and learning disability. Nat Genet 2006;38:1032-1037.

31. Sharp AJ, Hansen S, Selzer RR, Cheng Z, et al. Discovery of previously unidentified genomic disorders from the duplication architecture of the human genome. Nat Genet 2006;38:1038-1042.

32. Varela MC, Krepischi-Santos AC, Paz JA, Knijnenburg J, et al. A 17q21.31 microdeletion encompassing the MAPT gene in a mentally impaired patient. Cytogenet Genome Res 2006;114:89-92.

33. Ballif BC, Homor SA, Sulpizio SG, Lloyd RM, et al. Development of a high-density pericentromeric region $\mathrm{BAC}$ clone set for the detection and characterization of small supernumerary marker chromosomes by array CGH. Genet Med 2007;9:150-162. 